\title{
Synthesis, characterization and properties of ferroelectric rubidium hydrogen tartrate single crystals
}

\author{
C C DESAI and A H PATEL \\ Department of Physics, Sardar Patel University, Vallabh Vidyanagar 388 120, India \\ MS received 11 December 1987; revised 28 April 1988
}

\begin{abstract}
Rubidium hydrogen tartrate single crystals were grown in silica hydrogel. Controlled reaction was employed between tartaric acid and rubidium chloride solutions by slow diffusion process in the gel medium. Colourless transparent crystals were grown at room temperature. The average density of crystals measured pyknometrically was $2.263 \mathrm{~g} \mathrm{~cm}^{-3}$. Thermal stability of the materials was studied. Chemical etching was employed to determine the perfection of these crystals.
\end{abstract}

Keywords. Rubidium hydrogen tartrate single crystal; gel method; morphology; microhardness; etching; dislocations.

\section{Introduction}

Crystals of substances with high/low solubilities and a low thermal stability are difficult to grow from solutions. Gel media offers an efficient and simple technique for their growth. Recently a variety of crystals such as KDP or ADP (Lefaucheux et al 1982), $\mathrm{PbHPO}_{4}$ (Desai and Ramana 1987), $\mathrm{NaCl}, \mathrm{KCl}$ (Desai and Rai 1980) and also metallic crystals like $\mathrm{Ag}$ and $\mathrm{Cu}$ (Blank 1971) have been grown using gel medium.

Rubidium hydrogen tartrate (RHT) materials have attracted considerable attention on account of their ferroelectric and piezoelectric properties (Sonin and Strukov 1970). They are extensively used for controlling laser light (Pipree and Koblova 1967). The efficiency of RHT crystals in different modulation and defocussing systems is characterized by their electro-optical coefficients (Delfino et al 1979). Studies on the growth of RHT crystals in silica gels are however lacking and the present study attempts to fill this gap. The nucleation, growth, perfection and a few physico-chemical properties of RHT crystals are also reported.

\section{Experimental}

The technique (Halberstadt and Henisch 1968) consists of incorporating one reactant in the gelling mixture and later diffusing another reagent into the gel, leading to high supersaturation to initiate nucleation and crystal growth. To pure sodium metasilicate, required quantity of double-distilled water was added for getting the specific gravity $1.04 \mathrm{~g} \mathrm{~cm}^{-3}$. The required quantity of tartaric acid $(2.0 \mathrm{M})$ was added to form the gel medium. After the gels were set, the feed solution of rubidium chloride of varying strength $(0.5$ to $2.0 \mathrm{M})$ was added for crystallization. BDH AnalaR tartaric acid $(99.5 \%)$ and Fluka garantic rubidium chloride $(98 \%)$ were used for this purpose. No impurities were found (by optical absorption) in the reagents. The following chemical reaction was employed in the formation of single crystals of RHT 


$$
(\mathrm{CHOHCOOH})_{2}+\mathrm{RbCl} \rightarrow \mathrm{RbHC}_{4} \mathrm{H}_{4} \mathrm{O}_{6}+\mathrm{HCl} .
$$

Crystallization (size upto $16 \mathrm{~mm}$ ) was completed in 15 to 20 days. To determine the optimum growth conditions, a set of 40 experiments were carried out. The results were quite consistent and reproducible.

\section{Observations}

Various concentrations of both incorporated (tartaric acid) and supernatant ( $\mathrm{RbCl}$ ) solutions affected the size and quality of the grown RHT crystals. Table 1 illustrates the effect of concentration of the reactants and the incorporated solutions. The $\mathrm{pH}$ of gels was a predominant factor in the growth of RHT crystals. Figure 1(a) shows the crystals grown at $5.04 \mathrm{pH}$ which was found to be the optimum value for the growth. Single crystals grown above the optimum value $(5.35 \mathrm{pH})$ are shown in figure 1 (b). Figure 2 illustrates typical single RHT crystals grown in the present work. As the $\mathrm{pH}$ increased, the transparency of gel decreased. The crystals grown at higher $\mathrm{pH}$ values were opaque and poorly defined. This is due to contamination of crystals by silica gel.

To investigate the effect of gel, ageing gels were allowed to age for varying periods before adding the feed (rubidium chloride) solution. Rapid nucleation centres were observed at low gel age and less nucleation centres at high gel age. As shown by Henisch and Dennis (1967) gel ageing reduces the cell size and consequently the rate of diffusion of ions into the gel. At high gel age, RHT crystals of small size were grown at gel supernatant solution interface. It is observed that gel ageing has no pronounced effect on the quality of RHT crystals.

Table 1. Growth parameters for growing RHT single crystals.

\begin{tabular}{ccccl}
\hline $\begin{array}{c}\text { Concentration } \\
\text { of incorporated } \\
\text { tartaric acid } \\
\text { solution } \\
\text { (M) }\end{array}$ & $\begin{array}{c}\text { Concentration } \\
\text { of rubidium } \\
\text { chloride } \\
\text { solution (M) }\end{array}$ & $\begin{array}{c}\text { Growth } \\
\text { periods } \\
\text { (days) }\end{array}$ & $\begin{array}{c}\text { Size of grown } \\
\text { crystals } \\
\left(\mathrm{mm}^{3}\right)\end{array}$ & $\begin{array}{c}\text { Quality of } \\
\text { the grown } \\
\text { crystals }\end{array}$ \\
\hline 0.5 & 0.5 & 20 & $2 \times 1 \times 1$ & $\mathrm{p}$ \\
& 1.0 & 15 & $4 \times 2 \times 2$ & $\mathrm{p}$ \\
& 1.5 & 15 & $5 \times 2 \times 2$ & $\mathrm{~m}$ \\
& 2.0 & 15 & $7 \times 2 \times 2$ & $\mathrm{~g}$ \\
& 0.5 & 12 & $6 \times 3 \times 2$ & $\mathrm{p}$ \\
& 1.0 & 9 & $11 \times 4 \times 3$ & $\mathrm{~g}$ \\
& 1.5 & 9 & $16 \times 5 \times 3$ & $\mathrm{v.g}$ \\
& 2.0 & 9 & $8 \times 3 \times 2$ & $\mathrm{~m}$ \\
& 0.5 & 10 & $6 \times 4 \times 2$ & $\mathrm{~g}$ \\
& 1.0 & 10 & $7 \times 4 \times 2$ & $\mathrm{~m}$ \\
& 1.5 & 10 & $12 \times 4 \times 3$ & $\mathrm{v.g}$ \\
& 2.0 & 10 & $9 \times 5 \times 2$ & $\mathrm{p}$ \\
& 0.5 & 12 & $14 \times 3 \times 2$ & $\mathrm{~g}$ \\
& 1.0 & 12 & $10 \times 2 \times 2$ & $\mathrm{~m}$ \\
& 1.5 & 12 & $12 \times 4 \times 2$ & $\mathrm{p}$ \\
& 2.0 & 12 & $8 \times 3 \times 2$ & $\mathrm{p}$ \\
\hline
\end{tabular}

p. poor; m, medium; g, good; v.g., very good. 

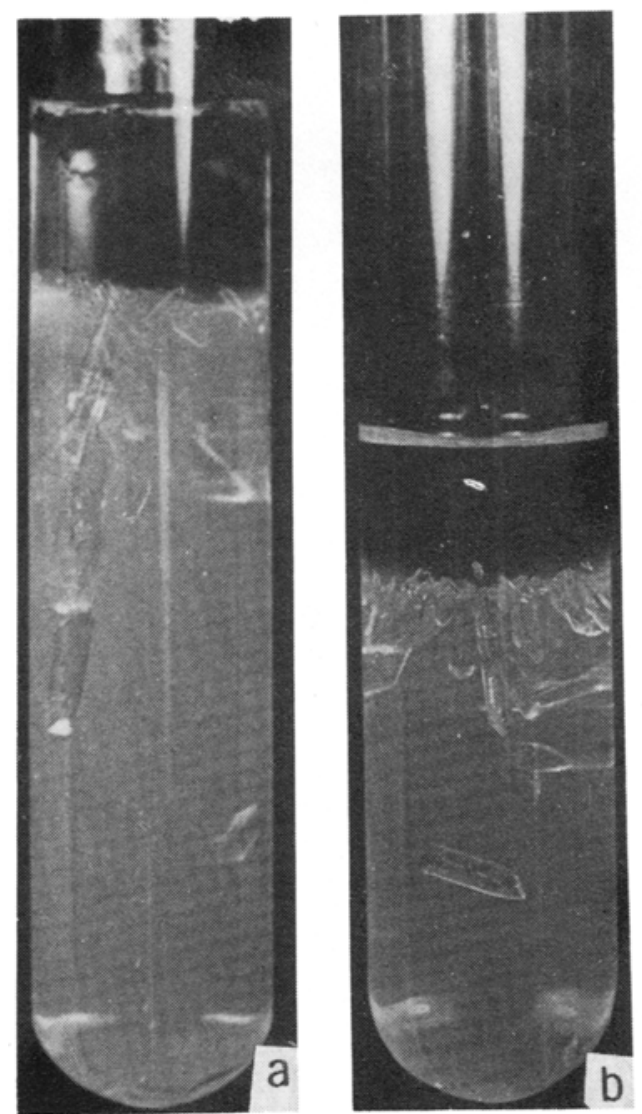

Figure 1. RHT single crystals grown at $\mathrm{pH}$ a. 5.04 and b. 5.35.

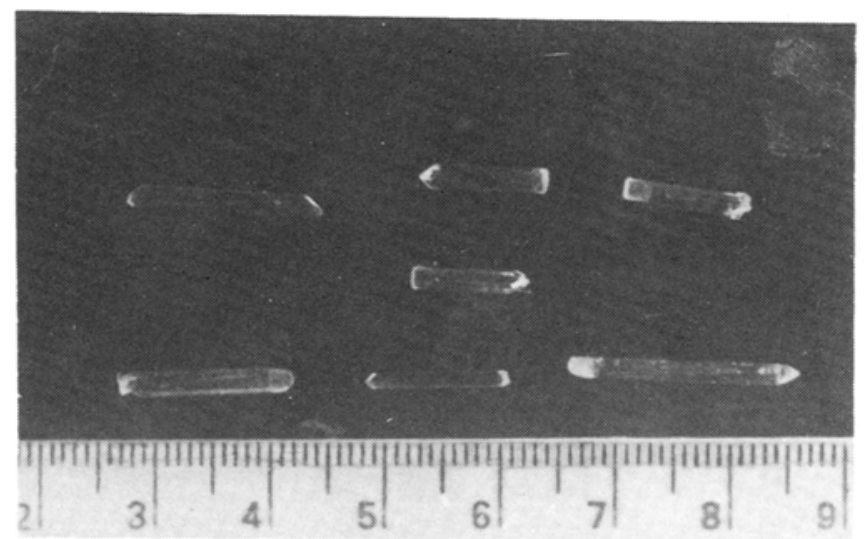

Figure 2. Typical single crystals of RHT grown in the present work.

\section{Characterization}

Chemical analysis, electron microprobe and EDAX analysis of the grown crystals 
confirmed the presence of carbon, hydrogen and rubidium ions. Calcium and chloride ions were identified as impurities. The percentage of carbon, rubidium and hydrogen determined by a Colman analyser were found to be $28.75,5.43$ and $8.38 \%$ respectively. The lattice parameters of these crystals determined by $\mathrm{X}$-ray rotation diffraction pattern (figure 3 ) showed excellent agreement with literature values $\left(a=7.621, b=10 \cdot 835, c=7.902 \AA, \alpha=\beta=\gamma=90^{\circ}\right.$, space group P2 $\left.2_{2} 2_{1}\right)$. Unit cell of the crystals contained four molecules. The average density of RHT crystals measured pyknometrically was $2.263 \mathrm{~g} \mathrm{~cm}^{-3}$. The microhardness determined by Vicker's hardness indentor for RHT was 12 VHN. Young modulus of the crystals determined by an Instron compression testing unit was $1.2 \times 10^{9} \mathrm{dynes} \mathrm{cm}^{-2}$ for [010]. These crystals exhibited cleavages parallel to the $\{010\}$ faces. By employing Faraday's gouy balance technique (Bates 1951), the molar susceptibility of RHT sample was measured and was $4.623 \times 10^{-3} \mathrm{emu}$. The positive values of magnetic susceptibility suggest that RHT are paramagnetic materials.

Figures 4(a) and (b) show the differential scanning calorimetric (DSC) and the thermogravimetric analyses (TGA) plots of RHT crystals. The endothermic peak at $473 \mathrm{~K}$ shows that these crystals get decomposed. This peak coincides with TGA plots and the corresponding weight loss due to decomposition is $10 \%$. The phase transition at $473 \mathrm{~K}$ to space group $\mathrm{P} 22_{1} 2_{1} 2_{1}$ is associated with the displacement of $(\mathrm{Rb})^{+}$ions whereby the mirror symmetry is destroyed.

\section{Morphology}

Some typical RHT crystals of different nature and needle crystals grown in the

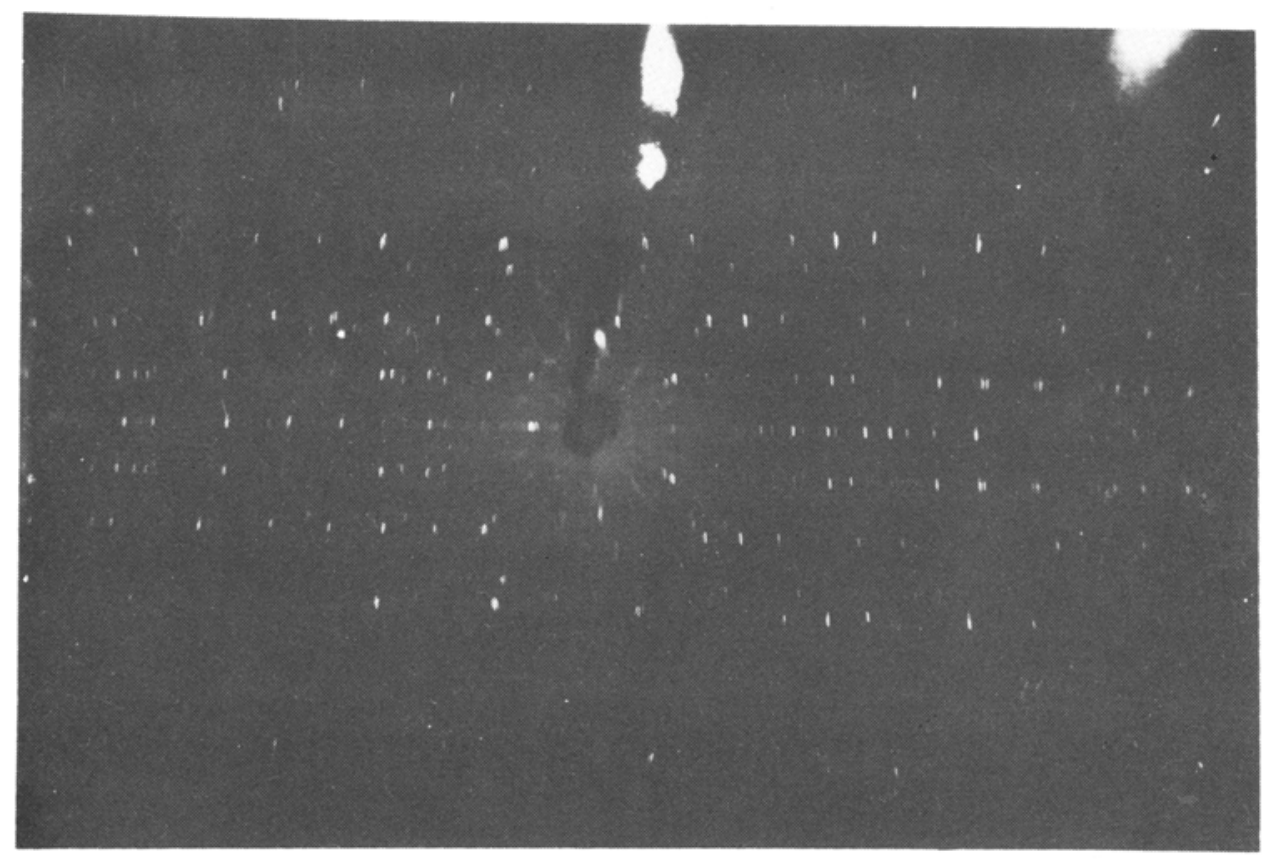

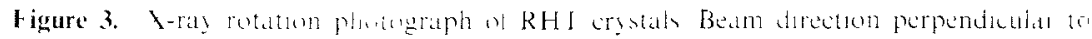
liv alv 
present work are shown in figure 5(a). Three types of morphologies of RHT crystals namely (i) disphenoidal tetrahedral crystals, (ii) orthorhombic disphenoidal crystals and (iii) prismatic needle-shaped crystals are observed. The morphology of these three types of crystals is depicted in figure 5(b). It is observed that the change in concentration of supernatant solution and $\mathrm{pH}$ has a remarkable effect on the morphology of the crystals. At $1.50 \mathrm{M}$ concentration of rubidium chloride solution, the crystals are in disphenoidal tetrahedral and orthorhombic morphologies while at $2.0 \mathrm{M}$ prismatic needle-shaped RHT crystals have been obtained.
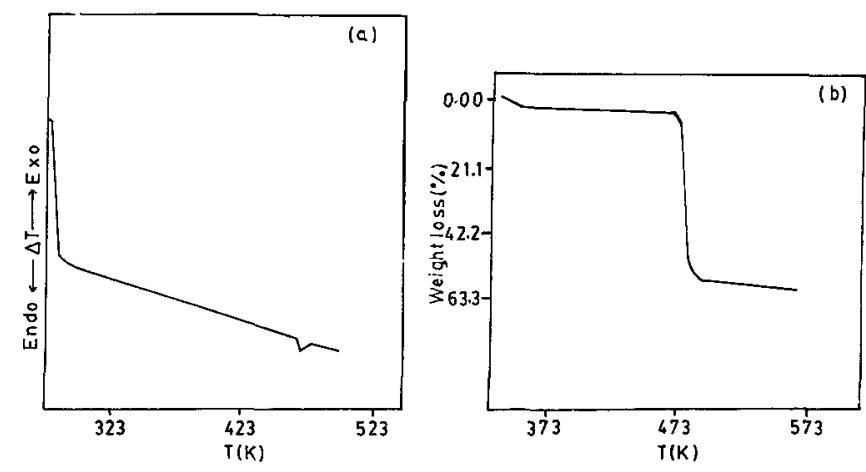

Figure 4. a. DSC and b. TGA curves of RHT crystals.

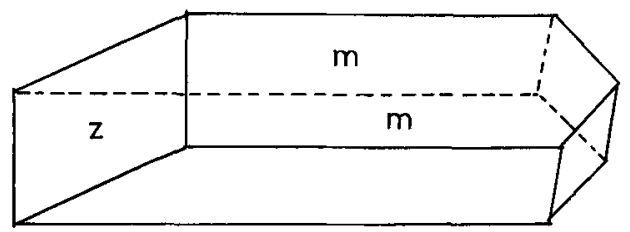

(i)

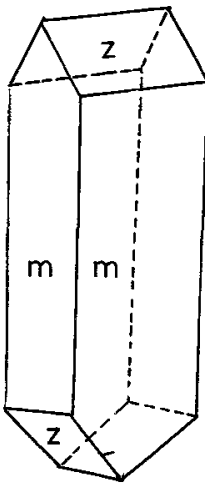

(iii)

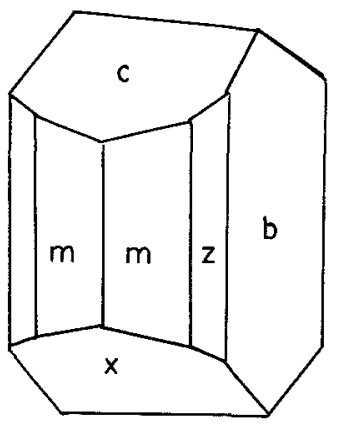

(ii)

Figure 5. a. Various morphologies of RHT crystals. (i) disphenoidal tetrahedral, (ii) orthorhombic disphenoidal and (iii) prismatic needleshaped. 


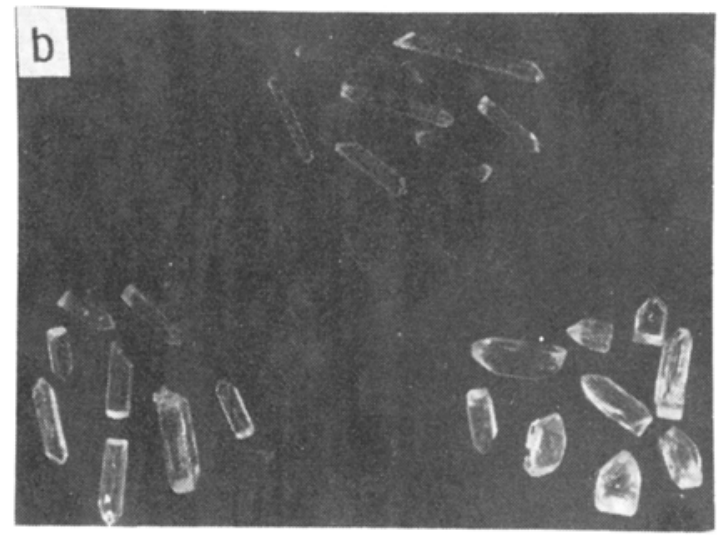

Figure 5. b. Schematic representatum of the different morphology of the grust erystals.

\section{Etching}

Perfection studies of the gel grown RHT crystals were made by the chemical etching technique. The $1.0 \mathrm{M}$ formic acid solution was used as an etchant which produced well-defined rectangular etch pits at the emergent sites of dislocations on the $(010)$ as-grown surfaces. The average density of dislocations was $10^{3} \mathrm{~cm}^{-2}$.

\section{Conclusions}

Silica hydrogels are a good media for growing good quality RHT. Colourless transparent RHT single crystals up to $16 \times 5 \times 3 \mathrm{~mm}^{3}$ in size were obtained. X-ray studies show that RHT crystals belong to the orthorhombic system at $300 \mathrm{~K}$ system and its space group was $\mathrm{P}_{2}, 2,2, \mathrm{Gel} \mathrm{pH}$ and concentration of feed solutions effect the crystal morphology. The magnetic susceptibility of RHT was $4.623 \times 10^{-3} \mathrm{emu}$. The positive value shows that RHT is a paramagnetic material. Etching experiments reveal the sites of dislocations and the crystalline perfection.

\section{Acknowledgements}

The authors thank Dr K C Desai (PAR Pharmaceutical Ltd.. New York). Prof. H L Patel and Mrs Shantaben Patel for keen interest and encouragement. One of the authors (CCD) thanks the University Grants Commission, New Delhi, for financial assistance.

\section{References}

Bates L F 1951 Wodern maynetism (Cambridge: University Press)

Blank Z 1971 J. Crystal. Growth 11255

Desai C C and Rai J L 1980 Krtst. Terh. 151115

Desai C C and Ramana M S V 1987 Crist. Re's. Technol. 22809

Delfino M. Loiacono G M. Usborne N N and Kustecky G 1979 J. Cryst. Growth 46241 
Halberstadt E S and Henisch H K 1968 J. Cryst. Growth 3/4 363

Henisch H K and Dennis J 1967 J. Electrochem. Soc. 114263

Lefaucheux F, Robert M C and Manghi E 1982 J. Cryst. Growth 56141

Pipree L V and Koblova M M 1967 Radio Eng. Electron. Phys. (Engl. Transl.) 12503

Sonin A S and Strukov B A 1970 Introduction to ferroelectrics (Moscow: High School Publ.) 